\title{
Jaaru: Efficiently Model Checking Persistent Memory Programs
}

\author{
Hamed Gorjiara \\ University of California, Irvine \\ USA \\ hgorjiar@uci.edu
}

\author{
Guoqing Harry Xu \\ University of California, Los Angeles \\ USA \\ harryxu@cs.ucla.edu
}

\author{
Brian Demsky \\ University of California, Irvine \\ USA \\ bdemsky@uci.edu
}

\begin{abstract}
Persistent memory (PM) technologies combine near DRAM performance with persistency and open the possibility of using one copy of a data structure as both a working copy and a persistent store of the data. Ensuring that these persistent data structures are crash consistent (i.e., power failures) is a major challenge. Stores to persistent memory are not immediately made persistent - they initially reside in processor cache and are only written to PM when a flush occurs due to space constraints or explicit flush instructions. It is more challenging to test crash consistency for PM than for disks given the PM's byte-addressability that leads to significantly more states.

We present Jaaru, a fully-automated and ultra-efficient model checker for PM programs. Key to Jaaru's efficiency is a new technique based on constraint refinement that can reduce the number of executions that must be explored by many orders of magnitude. This exploration technique effectively leverages commit stores, a common coding pattern, to reduce the model checking complexity from exponential in the length of program executions to quadratic. We have evaluated Jaaru with PMDK and RECIPE, and found 25 persistency bugs, 18 of which are new. Jaaru is also orders of magnitude more efficient than Yat, a model checker that eagerly explores all possible states.
\end{abstract}

\section{CCS CONCEPTS}

- Hardware $\rightarrow$ Memory and dense storage; • Software and its engineering $\rightarrow$ Software verification and validation.

\section{KEYWORDS}

Persistent Memory, Crash Consistency, Debugging, Testing

\section{ACM Reference Format:}

Hamed Gorjiara, Guoqing Harry Xu, and Brian Demsky. 2021. Jaaru: Efficiently Model Checking Persistent Memory Programs. In Proceedings of the 26th ACM International Conference on Architectural Support for Programming Languages and Operating Systems (ASPLOS '21), April 19-23, 2021, Virtual, USA. ACM, New York, NY, USA, 14 pages. https://doi.org/10.1145/3445814. 3446735

\section{INTRODUCTION}

Persistent memory (PM) technologies, such as phase change memory (PCM) [31, 51, 55], resistive random-access memory

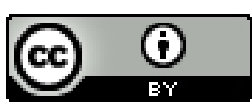

This work is licensed under a Creative Commons Attribution International 4.0 License. ASPLOS '21, April 19-23, 2021, Virtual, USA

(C) 2021 Copyright held by the owner/author(s)

ACM ISBN 978-1-4503-8317-2/21/04.

https://doi.org/10.1145/3445814.3446735
(RRAM) [50], Spin-Transfer Torque memory (STT-MRAM) [28], or 3D XPoint [11], promise to combine the performance and flexibility of DRAM with the persistency of flash storage. As commercially available in the Intel Optane memory product [12], persistent memory can interface with the processor via the memory bus, providing byte-addressable access for a program via regular store and load instructions. Such instructions bypass the OS kernel, offering a flexible and yet efficient interface to storage.

Persistent memory can potentially change the way programs manipulate data structures to achieve greater performance-with PM, programs can use a single copy of a data structure both as an in-memory working data structure and as a persistent store of the data, eliminating the serialization and deserialization process. Failures are a key challenge in realizing this approach-stores are not immediately written to persistent memory; they are initially written to the processor cache and the persistent memory is only eventually updated when the cache line is written back.

Modern processors provide special instructions to force cache lines to be written to persistent storage. Using these instructions correctly is challenging-it requires both subtle reasoning about the ordering of memory operations and attention to detail to not miss persisting any of the many stores a program may perform. Moreover, testing the correctness of persistent storage code w.r.t. failures is challenging. Exposing a bug requires that the machine fails at a specific instruction and depends on the state of the cache before the failure.

State of the art. The problem of PM consistency has received much attention. There is a line of recent work on testing/dynamically checking a PM program to find consistency-related bugs. XFDetector [36] uses a finite state machine to track the consistency and persistency of persistent data by implementing a shadow PM, and with the help of user-provided annotations to identify commit variables. XFDetector only shows violations of programming patterns for consistency and does not generate an execution that shows how the violation can actually lead to a bug. Moreover, it only supports scenarios in which a single failure occurs and ignores the possibility of the occurrence of failures in the post-failure execution. Different from XFDetector [36], PMTest [37] computes the persistency status of writes and ordering constraints between writes. Developers must annotate the code with checking rules to ensure that the code establishes the correct persistency and ordering properties. PMTest only executes the pre-failure portion of the program and thus does not test failure recovery, which may also contain bugs.

Pmemcheck [25] is a binary rewriting tool that checks how many stores were not made persistent and detects memory overwrites, redundant flushes, and unnecessary flushes [25]. Similar to PMTest, Pmemcheck also requires user annotations and only executes the pre-failure execution. 
These testing-based bug-finding tools suffer from two major drawbacks: (1) They need users to add extra annotations for various cache line flushing properties, which not only incur burdens on users but also are error-prone themselves. Consequently, if the developer misses an annotation or adds an incorrect annotation, the tool will have false negatives and miss real bugs or have false positives and report bugs that are not real. (2) Violations they report are with respect to design principles and may or may not correspond to actual bugs, e.g., certain tools report data has not been flushed. However, in some cases, the data may never be accessed in future executions. Thus, the absence of a flush is a false positive that does not represent a real bug. These drawbacks call for techniques such as model checking that can exhaustively explore states without needing manual effort and provide strong witnesses (e.g., executions) for bugs exposed.

Model checking has been used extensively in the systems community (e.g., EXPLODE [53], FiSC [54], or SAMC [33]) to find bugs in file/storage systems. However, there are several fundamental differences between the file system bug problem and persistent memory crash consistency problem that preclude direct application of existing model checkers in the PM setting: (1) disks have a fundamentally different programming interface than $\mathrm{PM}$ - updates to a disk block are only made upon making an explicit write request, (2) disks have a larger block size and therefore there are fewer possible states to enumerate, and (3) operating systems receive explicit notifications of when disk blocks are written. All of these factors combined indicate that the state space to be explored for model checking disks is significantly smaller than that for PM programs.

In fact, a recent technique Yat [29] attempts to use an eager model checking approach to enumerate all possible post-failure memory states for a PM program before it is aware of what parts of the state the post-failure execution will read from. Since the number of memory states that must be explored grows exponentially with the number of stores that have not been flushed to memory, Yat cannot scale. For example, consider the common scenario of code that allocates a cache line aligned array of $n$ 64-bit integers, initializes the data, and crashes right before flush operations for that array. This array spans $n / 8$ cache lines and the persistent memory copy of each cache line has 9 possible states (i.e., the initial value and the state after each of the 8 writes). Therefore, persistent memory has $9^{n / 8}$ possible states that Yat must explore.

Our approach. We develop Jaaru, a fully-automated and ultraefficient model checker for PM programs that achieves many ordersof-magnitude reductions in the number of states that must be explored, compared to eager techniques such as Yat. It does not require any user annotation; as a model checker, Jaaru exhaustively explores all possible states and can potentially find more bugs than testing-based techniques.

Key to Jaaru's efficiency is a constraint-refinement based technique that effectively leverages commit stores - a common programming practice in data structure implementations to drastically reduce the space of executions. We elaborate on this insight below.

As stated above, a major challenge in model checking PM programs is the enormous post-failure state space the model checker must explore - a store writes a value into the cache, and the value is not persisted until the cache line is flushed. However, when a failure occurs, it is unclear whether a cache line has been flushed yet, leading to a large number of possibilities that the model checker must explicitly enumerate.

To solve this problem, our major insight is that we can exhaustively explore all executions by enumerating only a subset of postfailure states using constraints on the time at which a cache line was previously flushed. A clflush or clflushopt instruction flushes a cache line, imposing a constraint on the possible values that a persistent variable can have after the failure. Jaaru builds such constraints during a pre-failure execution and refines them during a post-failure execution (see §3.1). Leveraging these constraints in partial order reduction $[15,56]$ enables Jaaru to explore exactly one post-failure state for each equivalence class of post-failure executions, defined by which pre-failure stores are read by post-failure loads.

To effectively leverage this insight, we made an observation that there are often many stores that have not been flushed out to persistent memory, PM programs often record in some fashion, using a commit store, whether data is in a consistent state (see § 3.2). For example, when adding a subtree to a node, the store of the node pointer to the subtree is a commit store. Post-failure PM programs then read from this commit store to determine whether data is consistent. This is a common practice in data structure implementations (1) because the information about consistency also provides a reference to where the data is stored (e.g., if the pointer from the node to subtree is null, the subtree is not persisted; otherwise, it can be found by following the pointer) and (2) for efficiency purposes. Such checks explicitly prevent the post-failure execution from accessing many unflushed stores (e.g., if the pointer is null, the program cannot access any data protected by the pointer).

This pattern offers an opportunity for us to not explicitly enumerate all possible states at a failure - lazily enumerating the stores read by the actual loads in the post-failure execution, as opposed to eagerly enumerating all of them, reduces the number of executions to be explored from exponential in the length of the program execution to linear (see §3.2). This observation leads to the lazy exploration approach used in Jaaru, which does not enumerate stores until loads are executed in the recovery code.

Note that leveraging such a programming pattern leads to efficiency, but has nothing to do with the thoroughness of the state search - Jaaru always exhaustively explores all the nondeterminism that arises from the persistency of cache lines. As a result, Jaaru does not generate any false positives or negatives - it reports all bugs w.r.t. an input and any bug it reports must be a real bug. For programs that do not obey such a programming idiom (e.g., the recovery code directly reads the data without checking consistency), Jaaru would not miss any bug, but it would certainly spend more time on state exploration. In practice, however, Jaaru is often still efficient because PM programs are extremely unlikely to read from many non-flushed cache lines.

Usage scenarios. Despite the aforementioned advantages, model checking is not a silver bullet for bug finding in PM programs. For example, even though Jaaru is orders of magnitude more efficient than existing model checkers such as Yat, Jaaru still needs to execute a program many times (e.g., between 24 and 891 in our experiments) to fully explore the state space, taking a large amount of time for 


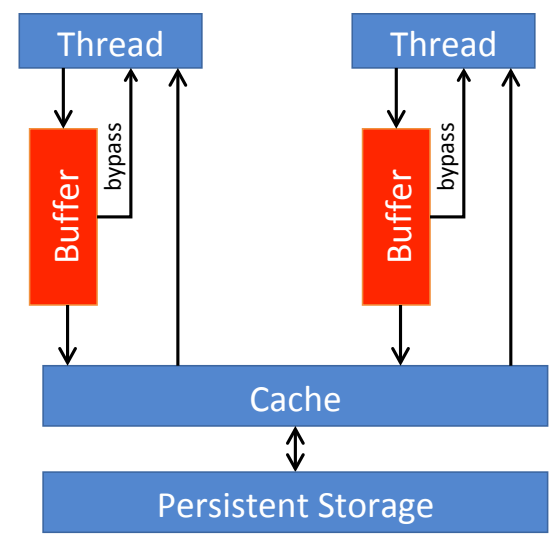

Figure 1: An x86-TSO storage system.

checking. Compared to testing tools such as PMTest and XFDetector, Jaaru is able to find more bugs, in a completely automated fashion. However, it has difficulty checking programs such as Redis that interact with the outside world and whose non-determinism from the network would require deterministic replay for a model checker to work. As such, the best use case for Jaaru is to exhaustively check widely-used libraries such as PMDK, finding as many potential bugs as possible before their release, while non-exhaustive tools such as PMTest and XFDetector can scalably check large programs and find bugs only when they are triggered in tests.

Summary of Results. We have implemented Jaaru which incorporates a full simulation of the underlying TSO memory model including support for store buffering, buffering flush operations, and buffering sfence operations. We evaluate Jaaru with PMDK [13] and RECIPE [32]: Jaaru is effective at finding persistency bugs in our benchmark set. Jaaru finds 18 new correctness bugs in extensively studied PM programs, while PMTest and XFDetector finds only 1 and 4 correctness bugs, respectively.

\section{OVERVIEW OF X86 PERSISTENT MEMORY STORAGE}

We next overview the Intel-x86 persistent storage system. We refer interested readers to the Px86 sim model in Raad et al. [43]. Figure 1 presents a graphical overview of the x86-TSO storage system. Each core/thread on $\mathrm{x} 86$ has a store buffer that buffers stores to the cache to hide the store latency. The store buffers implement bypassing - when a core performs a load, the core checks whether there is a store to the same address in its local store buffer. If so, it returns the value written by the most recent such store. Effectively, this allows the local core to observe the effect of a local store before that store becomes visible to other cores. The memory fence instruction mfence waits for the store buffer to be empty before future instructions can be executed. Locked RMW instructions also clear the store buffer before future instructions can be executed.

Stores in the store buffer are written to the cache in the order they were executed - they are written to the cache in a total order and all other threads/cores observe these stores in that same order. The cache is volatile - a power loss event will cause cached data that has not yet been written back to persistent storage to be lost. Under
Table 1: Summary of reordering constraints in the Px86 ${ }_{\text {sim }}$ model. A $\checkmark$ indicates that the order between the two instructions is preserved, $\mathrm{X} X$ indicates that the two instructions can be reordered, and a $C L$ indicates that the order is preserved only if they both operate on the same cache line. These constraints are also used in Raad et al. [43].

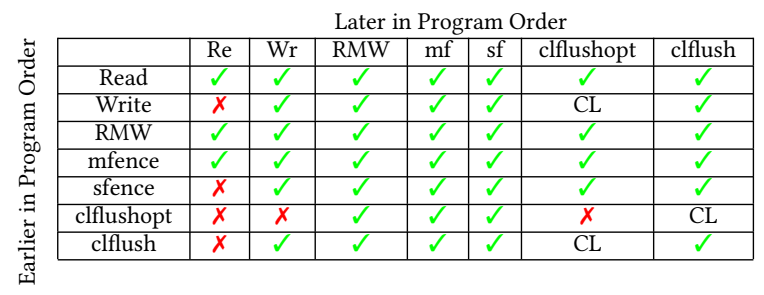

normal execution, cache lines are written back to main memory non-deterministically when the cache needs the space for other data. The x86 architecture provides instructions to force the cache to write data back to persistent storage. The three such instructions are: (1) the flush cache line instruction clflush that flushes a cache line, (2) the optimized flush cache line instruction clflushopt, and (3) the cache line write back instruction clwb.

A key difference between these instructions is how they can be reordered across other instructions. Table 1 summarizes the instruction ordering constraints for persistent storage on x86-TSO. The clflush instruction is inserted into the store buffer just like store instructions, and when it exits the store buffer it causes the cache line to be flushed to persistent memory. The clflushopt instruction is inserted into the store buffer also like store instructions, but it can be reordered across store instructions to other cache lines, clflush instructions to other cache lines, and other clflushopt instructions. The clflushopt instruction cannot be reordered across mfence or locked RMW instructions. The store fence instruction sfence also orders clflushopt instructions relative to clflush, clflushopt, clwb, and store instructions. The clwb instruction only writes back the contents of the cache line and does not evict it from the cache and thus has better performance. However, from a semantics perspective, the clwb instruction is identical to the clflushopt instruction [43], and thus we treat them identically in this paper.

\section{BASIC IDEAS}

Recall that prior work (e.g., Yat [29]) on model checking persistent memory programs eagerly enumerates all possible post-failure states of persistent memory. As the number of states grows exponentially with the amount of data that has not been flushed, this approach can easily have scalability problems. Such eager approaches will explore many post-failure states that yield identical post-failure executions in which the loads read from the same stores. Dynamic partial order reduction (DPOR) $[1,15,56]$ is a popular technique that can determine that these states produce the same execution, and instead explore the equivalent post-failure executions once. 


\subsection{Constraint-Refinement}

Traditional DPOR techniques do not consider the effect of cache line flushes and volatile memory. Naïve adaptation of these techniques in our setting would lead to the exploration of many states that are not possible due to the use of instructions such as clflush that explicitly flush cache lines.

To reduce search space, our first idea is to use clflush instructions to infer constraints on the last time each cache line was written back to persistent memory in a pre-failure execution and refine these constraints in a post-failure execution to narrow down when a cache line became persistent. For example, when a clflush instruction leaves the store buffer, it forces the cache line to be written back to persistent memory. That same cache line can later be written back to persistent memory due to space constraints in the cache. Hence, the clflush instruction essentially sets a constraint that the last time the corresponding cache line is written back to memory must be after the clflush instruction exits the store buffer.

Figure 2 illustrates the application of this idea on an execution prior to a failure. The program executes the instruction sequence on the left-hand side prior to the failure. The blue line shows the order that stores were written to the cache. Both variables $\mathrm{x}$ and $\mathrm{y}$ are located in the same cache line. After the program executes the stores $y=1$ and $x=2$, it performs a clflush instruction. This instruction flushes the cache line that holds $x$ and $y$ to persistent memory. At this point, Jaaru computes that the cache line for $x$ and $y$ was most recently flushed during the interval [clflush, $\infty$ ) as represented by the red line in Figure 2. After the clflush, the program performs the stores $y=3, x=4, y=5$, and $x=6$. Finally, power is lost and the program fails. The red interval indicates that when the machine is powered back up, the persistent storage may have the values 2, 4, and 6 for the variable $x$.

Note that there are constraints between the values for variable $x$ and those for $y$ since they share a cache line. For example, it is not possible for the post-failure state of the persistent memory to have $y=1$ and $x=6$, because the store $y=5$ is ordered between $y=1$ and $x=6$. To ensure that variables that share a cache line have consistent values, Jaaru refines these intervals using the values observed by loads during the recovery execution. Figure 3 shows a post-failure execution. This execution reads the value 4 from the variable $\mathrm{x}$. This tells us that the cache line must have been flushed some time after the store $x=4$ and before the store $x=6$. Thus, we can refine the interval for the most recent flush to be $[x=4, x=6$ ), which imposes a much tighter bound.

Since both variables $x$ and $y$ share the same cache line, reading the value 4 for $x$ constrains the set of values that we can read from $y$. In particular, since the last flush occurred some time during the interval from $x=4$ to $x=6$, we know that the cache line was flushed some time after the assignment $y=3$ and potentially after the assignment $y=5$. Therefore, if the post-failure execution reads from $y$, it could only read the value 3 or 5 . It could not read the value $y=1$, because the fact that the read from $x$ returned 4 tells us that the cache line was flushed after $y=1$ was overwritten.

Jaaru uses this refinement-based approach to simulate cache line flushes and lazily construct the state of persistent memory after the failure, eliminating the need to eagerly explore all (equivalence classes of) states.

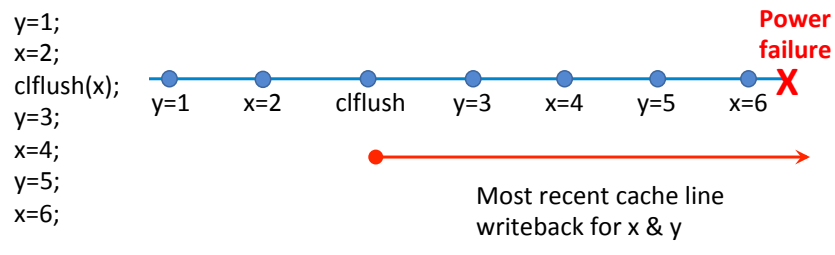

Figure 2: Pre-failure execution of a simple PM program. We assume that $x$ and $y$ reside on the same cache line. The blue line represents the total order in which stores are written to the cache. The red line shows the interval for the last time the cache line containing $x$ and $y$ may be written back to persistent memory.

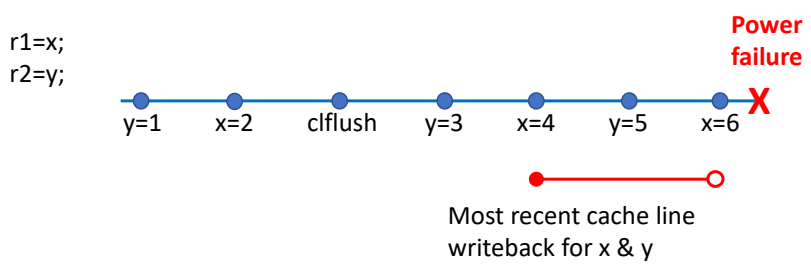

Figure 3: Post-failure (recovery) execution of the program that reads the value 4 from $x$. This refines the interval for the most recent writeback of the cache line to be between the store $x=4$ and the store $x=6$.

\subsection{Leveraging Commit Stores for Additional Efficiency}

Our constraint-refinement approach works well for PM programs because it effectively leverages commit stores to achieve efficiency. Commit stores are a rather common programming practice; in fact, all programs in our evaluation have such commit stores. To effectively leverage such stores, Jaaru does not eagerly enumerate all pre-failure stores; instead, Jaaru lazily enumerates a small subset of them that are actually read by a post-failure execution.

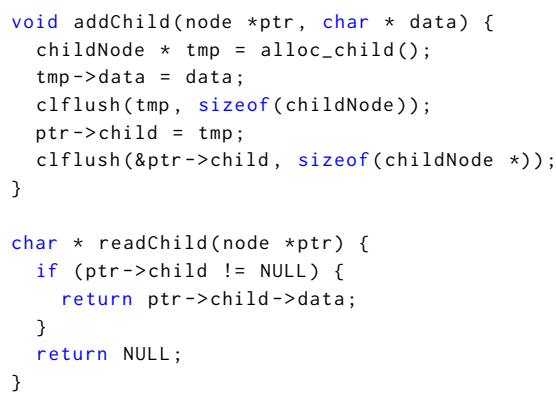

Figure 4: An example program with a commit store.

To illustrate, Figure 4 presents a simple program that uses a commit store. There are two methods here - method addChild that adds a child to store data and method readChild that returns a pointer to the data stored in the child. We first discuss the addChild 
method. The store at Line 3 writes a reference to the data field in the newly created child node. Next, the clflush instruction at Line 4 forces this write to persistent memory. Finally, the commit store at Line 5 makes the child node reachable from the data structure and the clflush at Line 6 makes the commit store persistent.

We next discuss the readChild method. The load at Line 10 checks whether the child field is non-null. If it is, then we know that (1) the clflush instruction at Line 6 completed and (2) the child node has been persisted and is safe to read in Line 11 .

To illustrate how Jaaru leverages this pattern for efficient state exploration, let us consider a client program that executes method addChild, fails, and then calls the readChild method during recovery. Jaaru injects failures in the execution of method addChild at three points: (1) immediately before the clflush instruction at Line 4, (2) immediately before the clflush instruction at Line 6, and (3) at the end of the execution of method addChild. Injecting failures at these three points is sufficient to explore all distinct program behaviors (see § 4). While Jaaru supports failure scenarios that involve crashes in the recovery routine, in this example we focus on a single failure for simplicity.

To inject a failure, Jaaru stops the execution at the failure point, resets volatile memory, and starts a new execution with the same persistent memory region. In the new execution, loads from persistent memory check the stores from the pre-failure execution to determine which values the program will read from.

Let us first consider the failure immediately before Line 4 . Since the clflush instruction has not executed, the write to the data field may not have been persisted. When the readChild method executes, it first reads the child field. Since the child field is null, it does not access the data field. Jaaru explores exactly one post-failure execution for this failure point.

Next, consider the failure immediately before Line 6 . The data field has been persisted by the first clflush instruction, but the write to the child field has not. Thus, when the post-failure execution reads from the child field, Jaaru observes that the interval for the most recent flush of the child field is $[0, \infty)$. Jaaru then explores two executions. In the first execution, the child field is null, and this execution has the same behavior as the previously explored execution. In the second execution, the child field is non-null and thus it reads the data field. Since the interval [clflush $4, \infty$ ) for the data field's cache line starts after the last write to the data field, the method returns the data field (clflush 4 denotes the clflush instruction at Line 4.).

Finally, consider the failure at the end of the execution of method addChild. At this point, both clflush instructions have executed. When the post-failure execution reads from the child field, Jaaru observes that the interval for the most recent flush of the child field is $\left[\right.$ clflush $\left._{6}, \infty\right)$. Therefore the load must see the value written to the child field and thus it reads the data field. Since the interval $\left[\right.$ clflush $\left._{6}, \infty\right)$ for the cache line of the data field starts after the last write to data, the method returns data.

To illustrate why such stores are useful, consider the following scenario. Suppose that method readChild accesses the data field of the child node without first checking the commit store in Line 5. If the addChild method crashes before the first clflush instruction, there would be two different potential post-failure states for the data field. If the child node has $n$ different cache lines that were

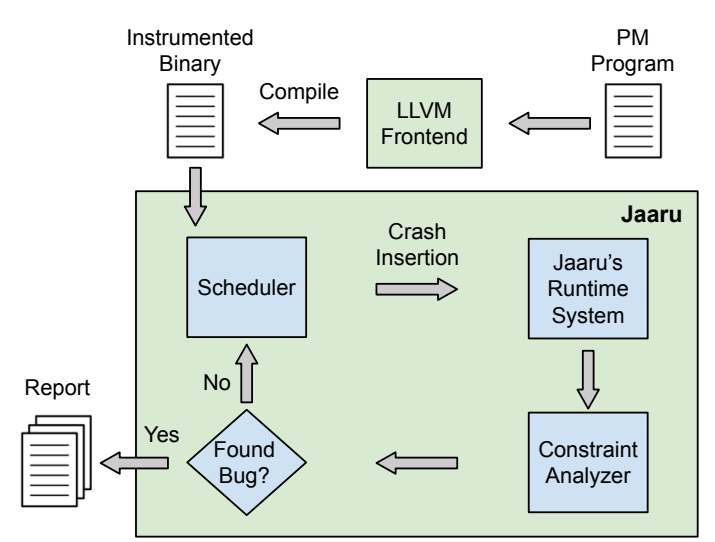

Figure 5: Jaaru system overview.

accessed in a similar manner, then the number of post-failure states would grow to be $O\left(2^{n}\right)$. If the post-failure code accesses all of the child's states, the model checker would have to explore $O\left(2^{n}\right)$ executions. The commit store limits the number of unflushed stores that the post-failure program execution reads from, and thus the executions Jaaru must explore.

The complexity of model checking programs that use commit stores like this example is $O\left(\mathrm{~m}^{2}\right)$ where $m$ is the length of the execution. We obtain this complexity because the number of failure injection points is $O(\mathrm{~m})$, the post-failure execution involves $O(\mathrm{~m})$ steps, and with commit stores, we explore two executions at each failure point - a first execution that reads from the commit store and a second execution that reads the value of the memory location before the commit store.

Note that prior techniques that eagerly explore all pre-failure stores cannot take advantage of such commit stores. The key difference between prior model checkers such as Yat and Jaaru is that Yat enumerates all possible states at the failure point before executing the post-failure code (thus with a complexity of $O\left(2^{n}\right)$ ) while Jaaru executes the post-failure code and lazily explores pre-failure stores that are actually read by the post-failure code.

\subsection{System Overview}

Jaaru uses an LLVM compiler pass to instrument both atomic and normal memory accesses along with fences and cache flush operations. The instrumented binary is then dynamically linked with the Jaaru library. Figure 5 presents an overview of Jaaru. A failure scenario involves multiple executions - the simplest failure scenario (a single failure) involves a pre-failure execution and a post-failure execution. To simulate a failure scenario, Jaaru keeps the information about each of the executions in the sequence that comprises the failure scenario. Figure 6 shows the exploration of a failure sequence composed of a pre-failure execution and the current post-failure execution.

Jaaru uses a fork-based approach to roll back executions to simulate failures and start new executions. In each execution, Jaaru records all of the stores that have been written to the cache and the clflush instructions that have taken effect (shown with the 


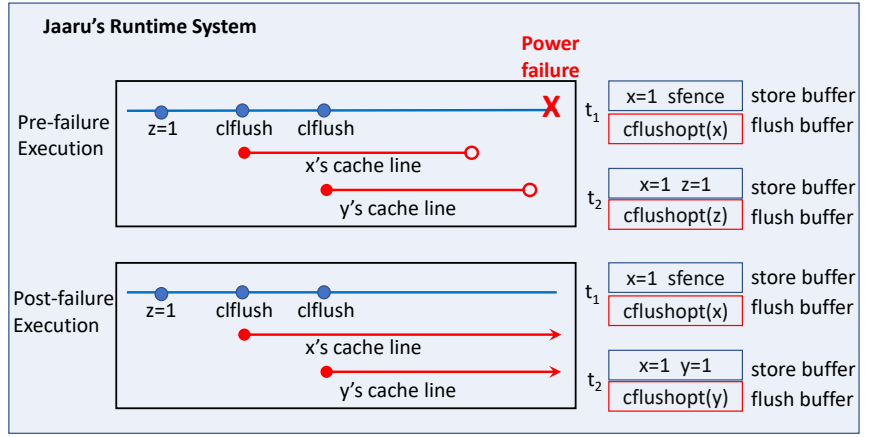

Figure 6: An example of Jaaru's runtime system.

blue lines). Jaaru also records a set of intervals for every flushed cache line to identify the time ranges of the most recent writes of each cache line into persistent memory (shown in the red lines). As shown earlier in the example, these intervals are used by the model checker to make decisions about the values of variables in the post-failure execution. The right side of each execution shows the thread-specific state Jaaru maintains - each thread has a local store buffer that simulates the processor's store buffer and a flush buffer that implements the reordering of clflushopt instructions (based on the constraints in Table 1).

\section{MODEL CHECKING ALGORITHM}

This section presents the model checking algorithm. We begin by presenting the following notations that we will use throughout the paper:

- We refer to an execution as $e$.

- A given failure scenario may involve a sequence of multiple executions ending in failures. We record this sequence of executions that have been executed on the persistent store using a stack, referred to as exec.

- Function top (exec) denotes the most recent execution (the current one) on the stack exec.

- Function $\operatorname{prev}(e)$ returns the execution that immediately precedes $e$ in exec.

- A global sequence number counter $\sigma_{\text {curr }}$ is used to assign increasing sequence numbers to stores, clflush, sfence instructions.

- Each store, clflush, and sfence instruction $i$ is assigned a sequence number $\sigma_{i}$. These numbers record the total order in which these instructions take effect in the cache.

- Each execution $e$ has a map e.getcacheline() that maps an address to an interval in which the cache line was most recently flushed to persistent memory in the exection $e$.

- Each execution $e$ has a map e.queue() that maps each address $a d d r$ to a sequence of tuples $\langle v a l, \sigma\rangle$ that record the values stored at the address and the sequence number $\sigma$ generated at the moment that value was stored.

- We denote a thread using $\tau \in \mathcal{T}$.

- Each thread $\tau$ has a store buffer $S_{\tau}$ that keeps a queue of store, clflush, and sfence operations that have not yet taken effect in the cache.
- Each thread $\tau$ has a cache line flush buffer $F_{\tau}$ that stores the set of clflushopt operations that have not yet flushed the cache line to persistent storage.

- We refer to the timestamp as t.

The Jaaru LLVM frontend instruments only memory operations and cache operations as those are the operations relevant to persistent storage. Jaaru implements a software simulation of those instructions with full support for the persistency semantics from the Px86 sim model [43]. The majority of PM-based tools have been developed for $\mathrm{x} 86$ since it provides the most advanced and mature architectural support for accessing persistent memory. By fully supporting x86 semantics, Jaaru satisfies the fast-growing need for a scalable and fast model checker to validate and test these programs. Although the current version of Jaaru is developed for $\mathrm{x} 86$, the primary idea behind it is not limited to $\mathrm{x} 86$ and could potentially be adapted to support other architectures such as ARM.

The TSO memory model separates the executions of stores, cache flush operations, and sfence operations into two phases: (1) the initial phase that often inserts an operation into a buffer and (2) the second phase that removes the instruction from the buffer and updates the state of the cache or persistent storage. We present our algorithm for each of the stages.

Executing instructions. Figure 7 presents our algorithm for the first phase of instruction execution, which inserts an instruction into each thread's local store buffer $S_{\tau}$. The mfence instruction waits until $S_{\tau}$ is empty and then clears the thread's flush buffer $F_{\tau}$.

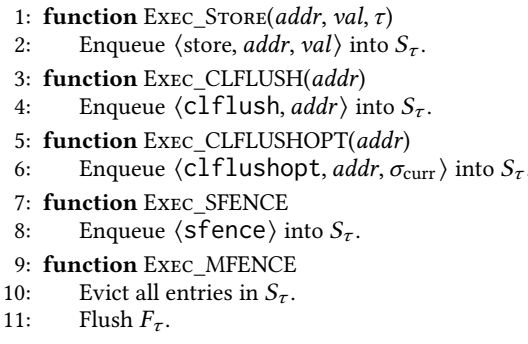

Figure 7: Algorithm for executing instructions.

Updating storage. The second phase occurs when the instruction leaves the store buffer. This phase updates the storage system. Figure 8 presents our algorithm for this phase. We have four different implementations of the EvICT_SB function for different types of instructions.

The Evict_SB( $\langle$ store, addr, val $\rangle)$ function handles store instructions. This function assigns a sequence number to each store. These sequence numbers enforce a total order over all writes to the cache. The function then moves the store to the queue of stores that records possible cache line values based upon the address it writes to. Finally, the function updates the timestamp $\left(t_{\tau, \operatorname{CacheID}(a d d r)}\right)$ for the most recent write to the cache line or clflush from this thread to be the store's sequence number.

The Evict_SB( $\langle$ clflush, $a d d r\rangle)$ function handles the cache line flush instruction clflush. The function first assigns a unique sequence number to the instruction. It then updates the lower bound of when the cache line was most recently flushed to be the sequence 


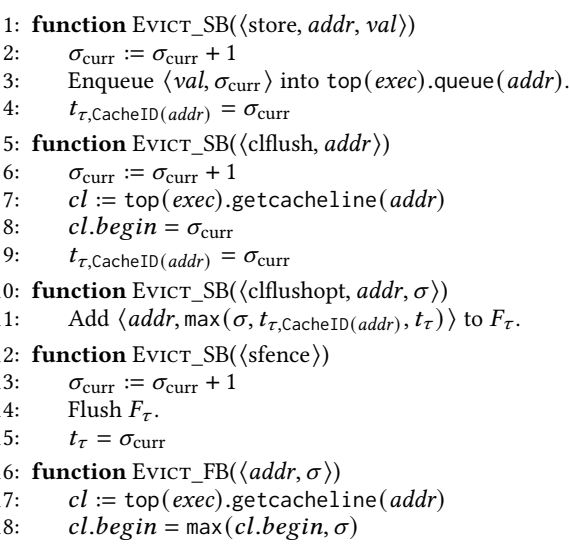

Figure 8: Algorithm for evicting store and flush buffers.

number for this particular flush operation. Finally, the function updates the timestamp for the most recent write to the cache line or clflush from this thread to be the store's sequence number.

The Evict_SB( $\langle$ clflushopt, $a d d r, \sigma\rangle)$ function handles the optimized cache line flush instruction clflushopt. The clflushopt instruction can be reordered with other clflushopt instructions, previous stores to other cache lines, clflush instructions to different cache lines, and later stores to any cache line. Support for reordering with previous operations is implemented by computing the maximum sequence number of the most recent instruction that the clflushopt cannot be reordered with. Support for reordering with later instructions is implemented by a flush buffer that is emptied when an instruction, which cannot be reordered with previous clflushopt instructions (i.e.,sfence, mfence, or RMW instructions), executes.

The Evict_SB $(\langle$ sfence $\rangle)$ function handles the store fence instruction sfence. This sfence instruction is ordered relative to all previous clflushopt instructions and thus it flushes the thread's flush buffer when it exits the thread's store buffer.

Finally, the Evict_FB $(\langle a d d r, \sigma\rangle)$ function handles clflushopt instructions when they are evicted from the flush buffer by an sfence, mfence, or RMW instruction. This function updates the lower bound of when the cache line was most recently flushed to be the sequence number $\sigma$ from the tuple in the flush buffer. Recall that this sequence number is the maximum of the following four values: (1) the current sequence number when the clflushopt instruction was first executed, (2) the sequence number of the most recent sfence instruction executed by the thread, (3) the sequence number of the most recent store to the same cache line executed by the same thread, or (4) the sequence number of the most recent clflush to the same cache line executed by the same thread.

Load operations. Figures 9 and 10 present our algorithm for loads. We split handling of loads into two functions: (1) the BuILDMAYREADFROM function that computes and returns a set of stores that a load may read from and (2) the DoREAD function that refines the cache line flush intervals once Jaaru has selected a specific store for the load to read from. Splitting the load handling into two components makes it straightforward to integrate loads into Jaaru's exploration.

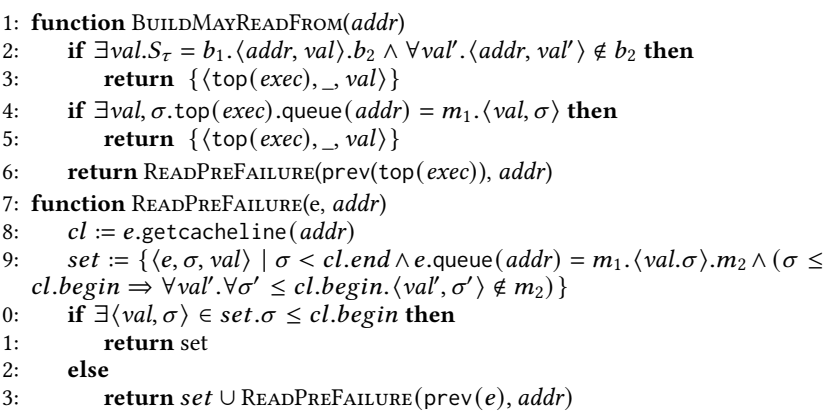

Figure 9: Algorithm for BuILdMAyReAdFrom.

We first discuss the BuILDMAYREAdFrom function in Figure 9. This function returns a set of tuples for each possible store that the load may read from. Each tuple contains the execution $e$ that performed the store, the sequence number $\sigma$ of the store, and the value val stored. We use when the store is from the current execution and thus does not have a sequence number that can be used to constrain when a cache line was last flushed in the previous execution.

Lines $2-3$ check whether there is a store to read from in the store buffer, and if so, returns the tuple for the newest such store. More precisely, the syntax $b_{1}$. $\langle a d d r, v a l\rangle . b_{2}$ represents the state of store buffer with $b_{1}$ being the oldest operations and $b_{2}$ being the newest operations. A load can read from a store $\langle a d d r, v a l\rangle$ in the store buffer if there are no newer stores to the same address.

Lines $4-5$ check whether there is a store in the current execution that has updated the cache. If so, they return the tuple for that store. More precisely, the syntax $m_{1} .\langle\mathrm{val}, \sigma\rangle$ represents the sequence of stores written to the cache with $m_{1}$ being the older operations. A load can read from a store $\langle\mathrm{val}, \sigma\rangle$ in the cache queue for an address if there are no newer stores to the same address. Otherwise, Line 6 invokes the READPREFAILURE function to compute potential stores from the executions before the most recent failure.

We next discuss the ReadPreFailure function in Figure 9. This function computes the set of stores from previous executions that a load may read from. Lines 8-9 compute the set of stores that would have been present on the cache line for the time range specified by the cache line's last flush interval. Line 10 checks whether there was a store performed before the earliest possible time for the cache line flush. If there is no such store, it is possible that the load has read from an earlier execution. In this case, the algorithm recursively calls READPREFAILURE on earlier executions and combines the set of stores from the current execution with those returned by the recursive call.

After the model checking algorithm has selected a store for the load to read from, it invokes the DoREAD function in Figure 10 to refine the most recent cache line flush intervals for previous executions. Line 2 checks whether the store is from the current execution. If so, there is no refinement to be performed and the function returns. Otherwise, it calls the function UpDATERANGES to refine the interval in which the last cache flush was performed. 


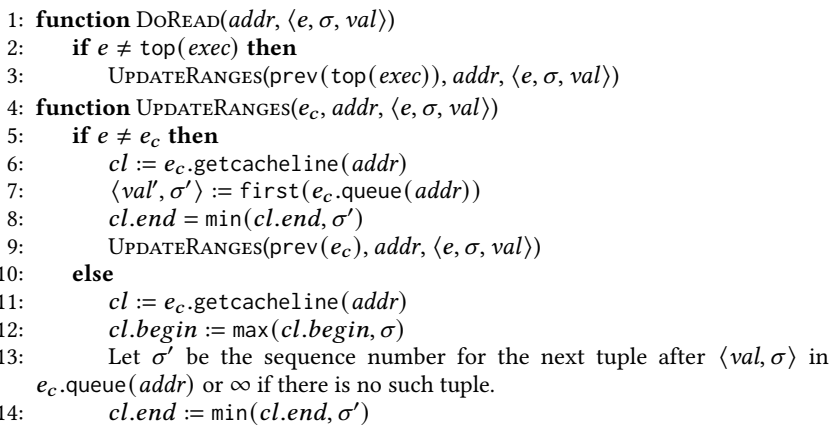

Figure 10: Algorithm for DoREAD.

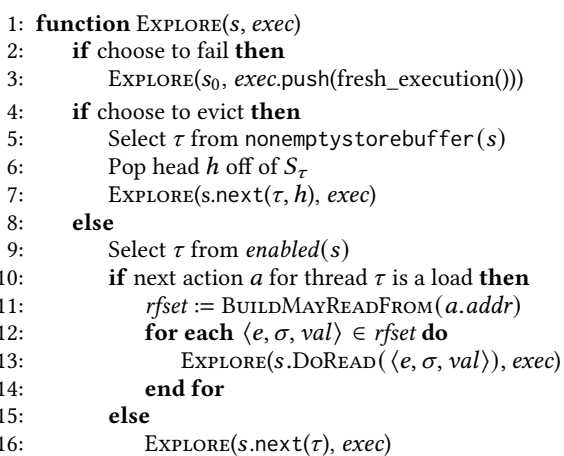

Figure 11: The main model checking Algorithm.

We next discuss the UpdateRANGe function. Line 5 checks whether the store is from the execution $e_{c}$. If not, Lines 6-9 refine the upper bound of the most recent flush interval to occur before the first store because the load reads from a store from a prior execution $e$ and thus we know that the current execution $e_{C}$ did not flush the cache line after performing a store. It then recursively calls the UPDATERANGE function on previous executions.

If the store is from the execution $e_{c}$, then Lines 11-14 refine the interval for the most recent cache line flush. The key insight is that the cache line must have been flushed after the store that the load reads from and before any subsequent stores.

Exploration algorithm. Finally, we present the core model checking algorithm. Figure 11 presents the ExPLORE function that implements Jaaru's exploration. The ExPLORE function takes in an execution $s$ and a stack of executions exec. Lines 2-3 inject failures and start new executions. Lines 4-8 decide whether to evict an entry from a thread's store buffer. Function next $(\tau, h)$ calls the appropriate Evict function from Figure 8. Line 9 selects the next thread to execute. Line 10 checks whether the thread's next operation is a load. If so, Lines 11-14 handle the load - we first call BuILDMAYREADFrom to compute the set of potential stores that the load may read from. The foreach loop then explores executions for each possible store that the load may read from.

If the thread's next operation is not a load, then Line 16 explores the next step. Function next $(\tau)$ computes the next step by calling the appropriate ExEc function from Figure 7.
Injecting failures. The natural points to inject failures are those immediately before operations that flush cache lines. The reason is that writes to the cache increase the set of possible post-failure executions while flushes decrease the set of possible post-failure executions. Thus, injecting failures at these points is sufficient to explore all program behaviors. Jaaru, therefore, injects failures at those points.

For long runs or scenarios in which multiple failures are injected, injecting failures before every flush can result in exploring many executions. Jaaru contains an optimization that skips injecting a failure if there have been no writes since the last injected failure. Jaaru can also support injecting failures into a post-failure execution (with a command line option). This option controls the maximum depth of the exec stack.

Locked RMW instructions. Locked (atomic) read-modify-write instructions include compare-and-swap (CAS), atomic exchange, and many atomic arithmetic instructions. On x86 these instructions also have fence-like semantics. They are equivalent to the atomic execution of the following sequence of instructions: mfence, load, store, and mfence. Jaaru implements them by atomically executing this sequence.

Mixed size accesses. $\mathrm{C}$ and $\mathrm{C}++$ programs may access fields using stores and loads with different widths. For example, a 32-bit integer field in a union may be initialized to 0 with a 64 -bit integer store and then read with a 32-bit integer load. We implement accesses that are larger than a byte as a sequence of byte accesses that are performed atomically. Thus, a 32-bit load is implemented as four 8-bit loads.

Checksum-based recovery. One approach to ensure data persistency is to write a checksum along with data - recovery code reads the checksum to verify that the data was persisted. Checksumbased recovery differs from other approaches in that the recovery code may read from a larger number of non-persisted stores. Jaaru provides special support that can exhaustively check programs that use checksum-based recovery without explicit flushes.

Debugging support. Jaaru can identify different types of bugs including missing fences, misordered flushes, missing flushes, and misordered stores that cause atomicity violations. The most common bug type we found was due to missing cache line flush instructions. Looking at the entire trace to understand a bug is not easy. Therefore, we extend Jaaru with additional functionality to help developers quickly determine why a program crashed.

Our observation is that a missing flush instruction effectively increases the number of pre-failure stores that a post-failure load may read from. Jaaru, therefore, contains optional support for flagging loads that can read from more than one store. To facilitate debugging, Jaaru prints out the load that can read from multiple stores, the source location of the load, each of the stores, their locations in the trace, and their source locations. Our experience shows that this information is very useful for quickly understanding missing flush instructions that cause the program to crash or loop.

Discussion. Existing DPOR algorithms [8, 15, 30, 45-47, 49] are not directly applicable in the setting of persistent memory. None of the traditional DPOR algorithms consider the effect of volatile memory such as crashes and cache flushes. For example, a crash makes 
pre-failure stores that were executed but not written to persistent memory completely disappear.

Jaaru can be viewed as implementing a form of dynamic partial order reduction that avoids exploring equivalent executions. Pre-failure executions that differ in when cache lines are flushed and thus generate different post-failure states can still yield the same post-failure executions if the post-failure executions never read from the memory locations that contain different values. Such cache line flushes can be viewed as commuting with the crash. Other cache line flushes make stores visible to post-failure loads and thus do not commute with the crash. Jaaru's constraint refinement algorithm lazily identifies non-commuting cache line operations during the post-failure execution and effectively explores reordering such cache line flushes.

Many PM programs are multi-threaded, creating the opportunity for concurrency bugs. Jaaru does not exhaustively explore all concurrent schedules and thus does not provide any guarantees that it will find concurrency bugs. However, since Jaaru controls the concurrent schedule and fully simulates the TSO memory model, as future work, it can be used to fuzz for concurrency bugs.

\section{EVALUATION}

In this section, we evaluate Jaaru's bug-finding capabilities and performance with a set of benchmarks. Our system configuration is reported in Table 2 .

Table 2: System configuration.

\begin{tabular}{|c|c|}
\hline CPU & 6-core $3.7 \mathrm{GHz}$ Intel i7-8700K processor \\
\hline Volatile Memory & 32GB DDR4, 2666MT/s \\
\hline Non-volatile Memory & Full Px86 sim $_{\text {sim }}$ semantics simulated (see $\left.\S 4\right)$ \\
\hline OS & Ubuntu Linux 18.04 \\
\hline Compiler & $\begin{array}{r}\text { gcc version } 7.5 .0 \text { opt level O3 } \\
\text { clang version } 11.0 .0 \text { opt level O3 }\end{array}$ \\
\hline
\end{tabular}

Our benchmarks. We have evaluated Jaaru on PMDK [13] and RECIPE [32]. PMDK is a library used extensively in prior work to evaluate bug-finding techniques [36, 37]. Both PMTest and XFDetector would require extra annotations to cover different behaviors of PMDK (e.g., PMTest requires the persistency order of every single variable to be defined by annotations). These annotations are on top of the normal assertions used to sanity check the program. However, Jaaru, as a model checker, can exhaustively explore the state space without the need to write any extra assertions other than the basic sanity checks that programs often have. For RECIPE, we were not able to run the P-HOT program because it did not compile with LLVM. All programs in the PMDK library have been used.

Memcached and Redis have both been ported to use PMDK and evaluated in prior work [36, 37]. Unfortunately, Memcached and Redis can only be executed as servers that interact with clients via sockets. Model checking a program that interacts with other programs requires support for deterministically replaying those socket interactions that the current version of Jaaru does not support. Jaaru could potentially be integrated with existing record-and-replay debugging frameworks to lift this limitation.

\subsection{Bug Detection}

We ran Jaaru over PMDK and RECIPE automatically to find bugs. The inputs are examples that come with these benchmark suites. We have not developed any new inputs ourselves. Jaaru has found a total of 25 bugs, of which 18 are new bugs that have not been reported before. Bugs that Jaaru can identify must have some visible manifestation - either a crash, e.g., segmentation fault, or an assertion failure in the program. Missing sanity checks in the program can result in silent data corruption where the program appears to recover successfully but has incorrect data.

We first discuss our experience with PMDK. Figure 12 reports the bugs we have found. For each bug, we list the program in which the bug was found. Note that the majority of these bugs are in the core libpmemobj library in PMDK and the examples merely have served as test cases for the library. For each bug, Figure 12 reports the symptoms of the bug, e.g., an assertion failure or illegal memory access. For many of these bugs, we have found that multiple failure injection points have led to the same symptom. These bugs may or may not be the same and to be conservative we report each such group of bugs as one bug. We have found 6 new bugs in the PMDK library - only bug \#2 was previously found by XFDetector [36]. Some of these bugs are not missing-flush bugs since the stores are followed by appropriate flush instructions, but atomicity violations in which partially completing updates leaves the data structures in inconsistent states. None of these 6 bugs was reported before (in either PMTest [37] or XFDetector [36]).

\begin{tabular}{|c|c|c|}
\hline$\#$ & Benchmark & Symptom \\
\hline 1 & Btree* $^{*}$ & Illegal memory access at btree_map.c:89 \\
2 & Btree $_{3}$ & Failed to open pool error \\
4 & Hashmap_atomic* $^{*}$ & Assertion failure at heap.c:533 \\
5 & CTree* $_{6}$ & Assertion failure at obj.c: 1523 \\
7 & Hashmap_atomic* $^{*}$ & Assertion failure at pmalloc.c:270 \\
7 & RBTree $^{*}$ & Illegal memory access at obj.c:1528 \\
\hline
\end{tabular}

Figure 12: Bugs found in PMDK. Bugs with $a$ * are new bugs. Only the second bug was reported before in XFDetector [36].

\begin{tabular}{|c|c|c|}
\hline \# & Benchmark & Type of Bug \\
\hline 1 & $\mathrm{CCEH}^{*}$ & Missing flush in $\mathrm{CCEH}$ constructor \\
\hline 2 & $\mathrm{CCEH}^{*}$ & Missing flush in CCEH constructor \\
\hline 3 & $\mathrm{CCEH}^{*}$ & Missing flush in CCEH constructor \\
\hline 4 & FAST_FAIR & Missing flush in header constructor \\
\hline 5 & FAST_FAIR & Missing flush in entry constructor \\
\hline 6 & FAST_FAIR* & Missing flush in btree constructor \\
\hline 7 & P-ART* & Use of non-persistent data structure in Epoch \\
\hline 8 & P-ART* & Missing flush in Tree constructor \\
\hline 9 & $\mathrm{P}-\mathrm{ART}^{*}$ & Use of non-persistent data structure for recovery \\
\hline 10 & P-BwTree* & GC crash leaves data structure in inconsistent state \\
\hline 11 & P-BwTree* & Missing flush of GC metadata pointer \\
\hline 12 & P-BwTree* & Missing flush of GC metadata \\
\hline 13 & P-BwTree* & Missing flush in AllocationMeta constructor \\
\hline 14 & P-BwTree* & Missing flush in BwTree constructor \\
\hline 15 & P-CLHT & Missing flush in clht constructor \\
\hline 16 & P-CLHT & Missing flush for hashtable object \\
\hline 17 & P-CLHT & Missing flush for hashtable array \\
\hline 18 & P-MassTree & Flushed referenced object instead of pointer \\
\hline
\end{tabular}

Figure 13: Bugs were found by Jaaru in every program of RECIPE. Bugs with $a$ * are new bugs. 
We next discuss results for the RECIPE benchmarks. We have found 12 new bugs in the RECIPE programs. Many programs contain multiple bugs. When Jaaru has found an execution that causes the program to crash (or loop) we have examined Jaaru's outputted trace and debugging information to understand the bug. Since these benchmarks are easier to understand than PMDK benchmarks, we have fixed the bug and used Jaaru to look for additional bugs. We continued this until the program executed correctly.

Figure 13 presents the bugs we have found. We confirmed that each bug caused the program to crash. Jaaru found bugs in every program. These bugs are primarily missing flush instructions in object constructors. All of the bugs can potentially corrupt a persistent data structure leading to data loss.

Many bugs are simple cases of forgetting to flush stores or mistakenly flushing the wrong memory location. However, we have found other kinds of bugs. In P-ART, the developer has used a vector data structure from tbb to track locks that must be unlocked in the recovery procedure. The bug is that tbb data structures do not persist across failures. In P-BwTree, Jaaru has found a logical error in the garbage collection (GC) algorithm in which failures during the GC can corrupt the GC data structures. This bug is an atomicity violation and not a case of missing flushes.

Comparing these results with the bugs found by PMTest [37] and XFDetector [36], Jaaru appears to have a stronger bug-finding ability than PMTest and XFDetector. For example, PMTest reported three new bugs and XFDetector reported four; several of these bugs were performance bugs. On the contrary, Jaaru found serious functional bugs that can corrupt data structures and lead to a crash or an assertion failure in the program. This is not surprising because Jaaru explores many more states than PMTest and XFDetector, which focus on a single execution.

Among the several bugs reported before, three were not found by Jaaru. We inspected those bugs and found it was because (1) two were performance bugs that are not our focus and (2) one was in the Redis code which we did not test. Jaaru could be extended to find performance bugs such as redundant cache flushes and fences. Jaaru Bug Reporting. We presented Jaaru and the bugs found by our tool to the authors of RECIPE and we received overall positive feedback. At the time of writing, 6 out of 18 bugs found by Jaaru were fixed by the developers of RECIPE. There were 6 bugs that were related to memory allocators and garbage collectors. The RECIPE developers did not fix the persistency bugs related to memory allocators because they believe these bugs need to be addressed by the memory allocators, which is not their focus. The remainder of the bugs were already fixed before our bug report.

\subsection{Performance}

Figure 14 presents the performance results for Jaaru on RECIPE benchmarks. Providing performance results for a model checker requires first fixing the bugs we have found so that Jaaru can run to completion and fully explore the state space of the program; otherwise, it would not make sense to report running time. We have spent much time fixing all the bugs we have found in RECIPE so that the model checker can fully explore these benchmarks. The bugs in the PMDK framework are more complicated and would take more time to fix, so we did not include our performance results for

\begin{tabular}{|l|c|c|c|c|}
\hline Benchmark & \#JExec. & JTime & \#FPoints & \#Yat Execs. \\
\hline CCEH & 891 & $14.51 \mathrm{~s}$ & 528 & $2.17 \times 10^{182}$ \\
FAST_FAIR & 170 & $1.48 \mathrm{~s}$ & 41 & $5.43 \times 10^{15}$ \\
P-ART & 174 & $1.86 \mathrm{~s}$ & 22 & $1.21 \times 10^{34}$ \\
P-BwTree & 71 & $0.79 \mathrm{~s}$ & 36 & $1.50 \times 10^{16}$ \\
P-CLHT & 25 & $1.59 \mathrm{~s}$ & 12 & $1.93 \times 10^{605}$ \\
P-Masstree & 24 & $0.17 \mathrm{~s}$ & 16 & $1.67 \times 10^{15}$ \\
\hline
\end{tabular}

Figure 14: Jaaru's state space reduction. Reported are the number of times Jaaru executes a program (JExec.), time Jaaru takes to finish exploration (JTime), number of failure injection points (FPoints), and the number of program executions Yat needs to eagerly explore pre-failure stores (Yat Execs.).

PMDK. Note that Jaaru is able to model check each RECIPE program in less than 15 seconds. We next discuss our evaluation of the state space reduction that Jaaru achieves on these programs, relative to an eager model checking approach such as that implemented in Yat [29]. Since Yat is not publicly available, we have calculated the number of legal post-failure states that Yat would have to explore. Figure 14 presents these results. Given the very large number of executions Yat would have to explore, it is unlikely to be feasible to exhaustively model check these realistic programs with Yat.

To better understand Jaaru's effectiveness, we compare the total number of executions with the number of failure injection points in the original execution. As shown in Figure 14, Jaaru only explores a few executions per failure injection point. The number of executions per failure injection point ranges from 1.5 to slightly less than 8 .

It does not make much sense to compare performance directly between Jaaru and non-exhaustive approaches such as PMTest and XFDetector, which detect bugs on single executions. However, as a reference, Jaaru incurs an overall slowdown of $736 \times$ per execution, which is on par with the overhead of XFDetector (i.e., from dozens of times to almost a thousand times as reported in the paper [36]). PMTest and Pmemcheck have much lower overhead $(1.69 \times$ and $22.3 \times$, respectively). This is because Jaaru fully simulates the $\mathrm{x} 86$ TSO persistency semantics while the other tools ignore the effects of store buffers.

Key Takeaway. Our results highlight the strengths and weaknesses of model checking: Jaaru finds more bugs without any user involvement, but cannot easily handle programs with complex interactions with the outside world. Jaaru is a good fit for checking library code that is usually small in size but has a large impact. Non-exhaustive tools such as PMTest and XFDetector should be used to check large programs such as Redis whose non-determinism from the network can give a model checker much trouble. It is also clear that the constraint refinement approach enables Jaaru to efficiently check these programs; without refinement, it would not be possible for a model checker to scale even to library code.

\section{RELATED WORK}

Bug/crash consistency detection. There exists a large body of work on testing [26, 29, 39, 52], checking [38, 44, 53, 54], and formally verifying $[9,10,48]$ file system implementations to find and eliminate crash consistency bugs. Fuzzing techniques such as Janus [52] and Hydra [26] mutate disk images and file operations 
to explore states of file system code. Using heuristics, B3 [39] employs a bounded testing technique to explore states in a bounded space. EXPLODE [53], FiSC [54], and SAMC [33] use model checkers to systematically explore states of a file system implementation. Although crash consistency bugs in file systems bear similarities with bugs in PM programs, they are fundamentally different in the access granularity as well as how writes are performed.

There is a recent line of work on checking/testing PM programs to find bugs. In particular, XFDetector [36] uses a finite state machine to track the consistency and persistency of persistent data PMTest [37] lets developers annotate a program with checking rules to infer the persistency status of writes and ordering constraints between writes. Pmemcheck [25] checks how many stores were not made persistent and detects memory overwrites using binary rewriting. Although these tools are able to find many bugs, none of these tools can systematically explore the state space. In particular, they simply check whether data is persisted appropriately. However, buggy data structures can have windows of vulnerability when crashes can cause failures even if all data is persisted and ordered. This motivates us to develop Jaaru, a model checker that can thoroughly explore states to find bugs.

Model checking. Model checking has been extensively studied. Stateless model checking techniques do not explicitly track which program states have been visited and instead focus on enumerating schedules [18-20, 40, 41]. To make model checking more efficient, researchers propose dynamic partial order reduction techniques [8, $15,30,45-47,49]$ that exploit state equivalence to reduce search space.

Recent work model-checks multi-threaded programs against the TSO and PSO memory models $[2,23,56]$ and the release-acquire fragment of $\mathrm{C} / \mathrm{C}++[3,5,14,27]$.

Model checking is also widely used to find bugs in systems code. Model checkers such as EXPLODE [53], FiSC [54], and SAMC [33] check file system code. However, directly applying these techniques would dictate enumerating all possible PM states, which is not feasible given that PM is byte-addressable and has orders of magnitude more states than a disk.

Yat [29] is an attempt to model check persistent memory. It injects failures before fence operations and eagerly enumerates all post-failure states to detect potential bugs.

Agamotto [42] finds bugs in persistent memory programs by using symbolic execution. It tracks the state of persistent memory objects and their corresponding cache lines in the program, i.e., whether the cache line is modified. Agamotto updates constraints on these states as the program runs and uses them to identify different types of persistency bugs including correctness, performance, and custom user-defined bugs. It uses a priority-based static analysis to steer program execution to program states that frequently modify PM. This approach can miss bugs because it only reasons about whether stores are made persistent and does not reason about the order that stores are made persistent.

Programming Models for PM. There is a great deal of work on building programming systems that allow developers to use PM in a reliable way without knowing the details of PM. For example, a line of work $[6,16,17,34]$ proposes to use (software or hardware) transactions to provide (failure and thread) atomicity. Another line of work $[4,7,22,24,35]$ advocates use of locks or synchronizationfree regions [21]. Jaaru is complementary to these approaches, it can be used to check the correctness of their implementation.

\section{CONCLUSION}

Jaaru is the first efficient model checker for persistent memory programs. Jaaru uses a constraint refinement-based approach that drastically reduces the number of executions that must be explored. Jaaru is the first tool to fully model the TSO persistent memory model. Our evaluation shows that Jaaru effectively finds bugs in our benchmark applications and that Jaaru reduces the number of executions that must be explored by several orders of magnitude.

\section{ACKNOWLEDGMENTS}

We would like to thank our shepherd, Jay Lorch, and the anonymous reviewers for their thorough and insightful comments that helped us substantially improve the paper. This work is supported by the National Science Foundation grants CNS-1703598, OAC-1740210, CNS-1703598, CNS-1763172, CCF-2006948, CNS-2007737, and CNS2006437, as well as ONR grants N00014-16-1-2913 and N00014-18$1-2037$.

\section{A ARTIFACT APPENDIX}

\section{A.1 Abstract}

This artifact contains a vagrant repository that downloads and compiles the source code for Jaaru, its companion compiler pass, and benchmarks. The artifact enables users to reproduce the bugs that are found by Jaaru in PMDK (i.e., Figure 11 of the paper) and RECIPE (i.e., Figure 12) as well as the performance results to compare Jaaru with Yat (i.e., Figure 13).

\section{A.2 Artifact Check-List (Meta-Information)}

- Algorithm: Lazy exhaustive model-checking

- Program: Jaaru

- Compilation: GCC 7.5.0 and Clang

- Binary: Instrumentation LLVM pass

- Data set: RECIPE and PMDK benchmarks

- Run-time environment: Any system that can run Vagrant

- Hardware: One 6 core $3.7 \mathrm{GHz}$ Intel i7 machine with 32 GB DDR4 memory

- Run-time state: Managed by our x86 simulator

- Execution: Automated by our tooling system

- Metrics: Crashing the program under test

- Output: Program crash for bugs. Logging performance measurement for executions.

- Experiments: Regenerating all bugs found by Jaaru. Reproducing performance results and comparing them with Yat (fully automated by our custom tooling)

- How much disk space required (approximately)?: $80 \mathrm{G}$

- How much time is needed to prepare workflow (approximately)?: 1 hour

- How much time is needed to complete experiments (approximately)?: About 20 mins

- Publicly available?: Yes. Open-source on GitHub

- Code licenses (if publicly available)?: GNU GENERAL PUBLIC LICENSE Version 2

- Data licenses (if publicly available)?: BSD-3-Clause and Apache License 2.0. 
- Workflow framework used?: Vagrant

- Archived (provide DOI)?:

https://doi.org/10.6084/m9.figshare.13392338

\section{A.3 Description}

Our workflow has four primary parts: (1) creating a virtual machine and installing dependencies needed to reproduce our results, (2) downloading the source code of Jaaru and the benchmarks and building them, (3) providing the parameters corresponding to each bug to reproduce the bugs, and (4) running the benchmarks to compare Jaaru with the naive exhaustive approach (i.e., Yat). After the experiment, the corresponding output files are generated for each bug and each performance measurement.

A.3.1 How to Access. All source code is open-source and available on GitHub. Our packaging requires cloning the vagrant system repository from https://github.com/uci-plrg/jaaru-vagrant. As described in the README.md file of the repository, you will need to install a VirtualBox VM and Vagrant on your machine. Then, the vagrant setup will install the required dependencies and download the source code of the tools from our git repository. Next, it builds each tool on the virtual machine.

A.3.2 Hardware Dependencies. Our tooling system and Jaaru have no special hardware dependencies and it can be running on any x86 machine with at least 32GB RAM and 4 cores.

A.3.3 Software Dependencies. To run our system, the following should be installed on the local machine:

- Linux (we tested on Ubuntu)

- Vagrant

- VirtualBox

- Vagrant-disksize plugin

A.3.4 Data Sets. To evaluate Jaaru, our tooling system downloads the source code of RECIPE and PMDK from our git repository. We forked a branch from the original source code of these benchmarks that don't contain our bug fixes. The tooling system automatically sets up and builds these benchmarks and runs them under Jaaru to identify bugs in them.

\section{A.4 Installation}

Please see the README.md file of the https://github.com/uci$\mathrm{plrg}$ /jaaru-vagrant repository, which contains a detailed step-bystep guide to setup Jaaru on a virtual machine. Then, our scripts automatically do the following:

(1) Install all the dependencies needed to install and evaluate Jaaru on different benchmarks.

(2) Check out the source code for LLVM, Jaaru, Jaaru's LLVM pass, RECIPE, and PMDK.

(3) Include Jaaru's LLVM pass to LLVM and building it

(4) Set up and build Jaaru with two different configurations (One for RECIPE that uses libvmemmalloc, and one for PMDK that uses libpmem APIs).

(5) Set up and building RECIPE (including CCEH, FAST_FAIR, P-ART, P-BwTree, P-CLHT, and P-Masstree benchmarks) and PMDK benchmarks.
(6) Generate three scripts in the home (or $\sim /$ ) directory of the virtual machine to generate the results.

Once the scripts are finished setting up the virtual machine and benchmarks, the user can use Jaaru on the virtual machine to further evaluate different benchmarks or regenerate our evaluation results.

\section{A.5 Experiment Workflow}

After setting up the virtual machine, the user can use 'vagrant ssh' to connect to the VM and use Jaaru. The detailed instructions to run the suggested workflow is included in the README.md file of https://github.com/uci-plrg/jaaru-vagrant repository. There are three scripts in the home directory of the virtual machine that user can run:

recipe-perf.sh : It runs the RECIPE benchmarks using Jaaru and gathers measurements to compare Jaaru against Yat. For each benchmark, the corresponding log file is generated in $\sim /$ results/recipeperformance.

recipe-bugs.sh : It runs the RECIPE benchmarks using Jaaru and sets the corresponding parameters to reproduce each bug. For each bug, the corresponding log file is generated in $\sim /$ results/recipe-bugs. pmdk-bugs.sh : It runs PMDK benchmarks by using Jaaru and set the corresponding parameters to reproduce each bug. For each bug, the corresponding log file is generated in $\sim /$ results/pmdk-bugs.

In our tooling system, the timeout is used in both recipe-bugs.sh and pmdk-bugs.sh scripts to recover from segmentation fault. The timeout needs to be adjusted if the user uses a slower machine.

\section{A.6 Evaluation and Expected Result}

After successfully running the experiment using our scripts, the results directory is generated in the home directory. This directory contains the following results:

A.6.1 RECIPE. Performance Results: For each RECIPE benchmark, there is a-Performance file in the $\sim /$ results/recipe-performance directory (for a total of 6 files). These files contain the performance information corresponding to Figure 13.

Bugs: There are 18 files in /results/recipe-bugs directory. Each file contains the corresponding logs for the bug that Jaaru found. Figure 15 contains information about how Jaaru identified each bug correspond to Figure 12.

A.6.2 PMDK. There are 7 files in $\sim /$ results/pmdk-bugs directory. Each file contains the corresponding logs for the bug that Jaaru found. Figure 16 contains information about how Jaaru identified each bug correspond to Figure 11.

\section{A.7 Experiment Customization}

The experiment workflow can be customized to install and run everything on the local machine instead of the virtual machine. To set up everything locally, download data/setup.sh script from the https://github.com/uci-plrg/jaaru-vagrant repository in the home directory of your local machine and run the script after installing the dependencies. 


\begin{tabular}{|c|c|c|}
\hline$\#$ & Bug ID & Cause of Bug \\
\hline 1 & CCEH-1 & Getting stuck in an infinite loop \\
2 & CCEH-2 & Segmentation fault in the program \\
3 & CCEH-3 & Segmentation fault in the program \\
4 & FAST_FAIR-1 & Segmentation fault in the program \\
5 & FAST_FAIR-2 & Segmentation fault in the program \\
6 & FAST_FAIR-3 & Segmentation fault in the program \\
7 & P-ART-1 & Segmentation fault in the program \\
8 & P-ART-2 & Illegal memory access in the program \\
9 & P-ART-3 & Getting stuck in an infinite loop \\
10 & P-BwTree-1 & Segmentation fault in the program \\
11 & P-BwTree-2 & Segmentation fault in the program \\
12 & P-BwTree-3 & Segmentation fault in the program \\
13 & P-BwTree-4 & Segmentation fault in the program \\
14 & P-BwTree-5 & Segmentation fault in the program \\
15 & P-CLHT-1 & Illegal memory access in the program \\
16 & P-CLHT-2 & Illegal memory access in the program \\
17 & P-CLHT-3 & Getting stuck in an infinite loop \\
18 & P-MassTree-1 & Illegal memory access in the program \\
\hline
\end{tabular}

Figure 15: More information about the bugs that are found by Jaaru in RECIPE benchmarks.

\begin{tabular}{|c|c|c|}
\hline$\#$ & Benchmark Found & Symptom \\
\hline 1 & Btree* $^{*}$ & Illegal memory access at btree_map.c:89 \\
2 & Btree & Failed to open pool error \\
3 & Hashmap_atomic* $^{*}$ & Assertion failure at heap.c:533 \\
4 & CTree* $^{*}$ & Assertion failure at obj.c:1523 \\
5 & Hashmap_atomic* $^{*}$ & Assertion failure at pmalloc.c:270 \\
6 & Hashmap_tx* $_{7}^{*}$ & Illegal memory access at obj.c:1528 \\
7 & RBTree* $^{*}$ & Assertion failure at tx.c:1678 \\
\hline
\end{tabular}

Figure 16: More information about the bugs that are found by Jaaru in PMDK benchmarks.

\section{A.8 Notes}

Note that the performance results generated for RECIPE can be different from the numbers that are reported in the paper since there is non-determinism in scheduling threads; when stores, flushes, and fences leave the store buffer; and memory alignment in the malloc procedure. This non-determinism can possibly impact on the type of bugs reported in Figure 15 and Figure 16 for RECIPE and PMDK benchmarks. Also, for some bugs, the segmentation fault (or assertion failure) occurs in Jaaru code. This is caused by illegal memory access by the program under test.

\section{REFERENCES}

[1] Parosh Abdulla, Stavros Aronis, Bengt Jonsson, and Konstantinos Sagonas. Optimal dynamic partial order reduction. In Proceedings of the 2014 Symposium on Principles of Programming Languages, POPL '14, pages 373-384, 2014.

[2] Parosh Aziz Abdulla, Stavros Aronis, Mohamed Faouzi Atig, Bengt Jonsson, Carl Leonardsson, and Konstantinos Sagonas. Stateless model checking for TSO and PSO. In Proceedings of the 21st International Conference on Tools and Algorithms for the Construction and Analysis of Systems, pages 353-367, 2015.

[3] Parosh Aziz Abdulla, Mohamed Faouzi Atig, Bengt Jonsson, and Tuan Phong Ngo. Optimal stateless model checking under the release-acquire semantics. Proceedings of the ACM on Programming Languages, October 2018.

[4] Hans-J. Boehm and Dhruva R. Chakrabarti. Persistence Programming Models for Non-volatile Memory. In Proceedings of the 2016 ACM SIGPLAN International Symposium on Memory Management, ISMM 2016, pages 55-67, 2016.

[5] Sebastian Burckhardt, Rajeev Alur, and Milo M. K. Martin. CheckFence: Checking consistency of concurrent data types on relaxed memory models. In Proceedings of the 28th ACM SIGPLAN Conference on Programming Language Design and Implementation, PLDI '07, pages 12-21, 2007.

[6] Daniel Castro, Paolo Romano, and João Barreto. Hardware transactional memory meets memory persistency. In 2018 IEEE International Parallel and Distributed Processing Symposium, IPDPS '18, pages 368-377, 2018.

[7] Dhruva R. Chakrabarti, Hans-J. Boehm, and Kumud Bhandari. Atlas: Leveraging Locks for Non-volatile Memory Consistency. In Proceedings of the 2014 ACM International Conference on Object Oriented Programming Systems Languages and
Applications, OOPSLA '14, pages 433-452, 2014

[8] Krishnendu Chatterjee, Andreas Pavlogiannis, and Viktor Toman. Value-centric dynamic partial order reduction. Proceeding of the ACM on Programming Languages, 3(OOPSLA), October 2019.

[9] Haogang Chen, Tej Chajed, Alex Konradi, Stephanie Wang, Atalay İleri, Adam Chlipala, M. Frans Kaashoek, and Nickolai Zeldovich. Verifying a highperformance crash-safe file system using a tree specification. In Proceedings of the 26th Symposium on Operating Systems Principles, SOSP '17, pages 270-286, 2017.

[10] Haogang Chen, Daniel Ziegler, Tej Chajed, Adam Chlipala, M. Frans Kaashoek, and Nickolai Zeldovich. Using crash Hoare logic for certifying the FSCQ file system. In Proceedings of the 25th ACM Symposium on Operating Systems Principles, SOSP '15, pages 18-37, 2015.

[11] Intel Corporation. 3D XPoint ${ }^{T M}$ : A breakthrough in non-volatile memory technology. https:/www.intel.com/content/www/us/en/architecture-andtechnology/intel-micron-3d-xpoint-webcast.html, 2018.

[12] Intel Corporation. Intel announces broadest product portfolio for moving, storing and processing data. https://newsroom.intel.com/news-releases/intel-datacentric-launch/\#gs.d3t61g, April 2019.

[13] Intel Corporation. Persistent memory development kit. https://pmem.io/pmdk/, 2020.

[14] Brian Demsky and Patrick Lam. SATCheck: SAT-directed stateless model checking for SC and TSO. In Proceedings of the 2015 ACM SIGPLAN International Conference on Object-Oriented Programming, Systems, Languages, and Applications, OOPSLA 2015, pages 20-36, October 2015.

[15] Cormac Flanagan and Patrice Godefroid. Dynamic partial-order reduction for model checking software. In Proceedings of the 32nd ACM SIGPLAN-SIGACT Symposium on Principles of Programming Languages, POPL '05, pages 110-121, 2005.

[16] Kaan Genç, Michael D. Bond, and Guoqing Harry Xu. Crafty: Efficient, HTMCompatible Persistent Transactions. In Proceedings of the 41st ACM SIGPLAN Conference on Programming Language Design and Implementation, PLDI 2020, pages 59-74, 2020.

[17] Ellis Giles, Kshitij Doshi, and Peter Varman. Continuous Checkpointing of HTM Transactions in NVM. In Proceedings of the 2017 ACM SIGPLAN International Symposium on Memory Management, ISMM '17, pages 70-81, 2017.

[18] Patrice Godefroid. Partial-Order Methods for the Verification of Concurrent Systems: An Approach to the State-Explosion Problem. Springer-Verlag, Berlin, Heidelberg, 1996.

[19] Patrice Godefroid. Model checking for programming languages using VeriSoft. In Proceedings of the 24th ACM SIGPLAN-SIGACT Symposium on Principles of Programming Languages, POPL '97, pages 174-186, 1997.

[20] Patrice Godefroid. Software model checking: The VeriSoft approach. Formal Methods in System Design, 26(2):77-101, 2005.

[21] Vaibhav Gogte, Stephan Diestelhorst, William Wang, Satish Narayanasamy, Peter M. Chen, and Thomas F. Wenisch. Persistency for Synchronization-Free Regions. In Proceedings of the 39th ACM SIGPLAN Conference on Programming Language Design and Implementation, PLDI '18, pages 46-61, 2018.

[22] Terry Ching-Hsiang Hsu, Helge Brügner, Indrajit Roy, Kimberly Keeton, and Patrick Eugster. NVthreads: Practical Persistence for Multi-threaded Applications. In Proceedings of the 12th European Conference on Computer Systems, EuroSys '17, pages 468-482, 2017.

[23] Shiyou Huang and Jeff Huang. Maximal causality reduction for TSO and PSO. In Proceedings of the 2016 ACM SIGPLAN International Conference on Object-Oriented Programming, Systems, Languages, and Applications, OOPSLA '16, pages 447-461, 2016.

[24] Joseph Izraelevitz, Terence Kelly, and Aasheesh Kolli. Failure-Atomic Persistent Memory Updates via JUSTDO Logging. In Proceedings of the 21st International Conference on Architectural Support for Programming Languages and Operating Systems, ASPLOS '16, pages 427-442, 2016.

[25] Tomasz Kapela. An introduction to pmemcheck (part 1) - basics. https://pmem. io/2015/07/17/pmemcheck-basic.html, July 2015.

[26] Seulbae Kim, Meng Xu, Sanidhya Kashyap, Jungyeon Yoon, Wen Xu, and Taesoo Kim. Finding semantic bugs in file systems with an extensible fuzzing framework. In Proceedings of the 27th ACM Symposium on Operating Systems Principles, SOSP '19, pages 147-161, 2019.

[27] Michalis Kokologiannakis, Ori Lahav, Konstantinos Sagonas, and Viktor Vafeiadis. Effective stateless model checking for $\mathrm{C} / \mathrm{C}++$ concurrency. Proceedings of the ACM on Programming Languages, 2(POPL), December 2017.

[28] Emre Kultursay, Mahmut Kandemir, Anand Sivasubramaniam, and Onur Mutlu. Evaluating STT-RAM as an energy-efficient main memory alternative. In IEEE International Symposium on Performance Analysis of Systems and Software, ISPASS '13, pages 256-267, 2013.

[29] Philip Lantz, Subramanya Dulloor, Sanjay Kumar, Rajesh Sankaran, and Jeff Jackson. Yat: A validation framework for persistent memory software. In Proceedings of the 2014 USENIX Annual Technical Conference, pages 433-438, Philadelphia, PA, June 2014. USENIX Association. 
[30] Steven Lauterburg, Rajesh K Karmani, Darko Marinov, and Gul Agha. Evaluating ordering heuristics for dynamic partial-order reduction techniques. In Inter national Conference on Fundamental Approaches to Software Engineering, pages 308-322. Springer, 2010

[31] Benjamin C. Lee, Engin Ipek, Onur Mutlu, and Doug Burger. Architecting phase change memory as a scalable DRAM alternative. In Proceedings of the 36th Annual International Symposium on Computer Architecture, ISCA '09, pages 2-13, 2009.

[32] Se Kwon Lee, Jayashree Mohan, Sanidhya Kashyap, Taesoo Kim, and Vijay Chidambaram. Recipe: Converting concurrent DRAM indexes to persistent-memory indexes. In Proceedings of the 27th ACM Symposium on Operating Systems Principles, SOSP '19, pages 462-477, New York, NY, USA, 2019. Association for Computing Machinery.

[33] Tanakorn Leesatapornwongsa, Mingzhe Hao, Pallavi Joshi, Jeffrey F. Lukman, and Haryadi S. Gunawi. SAMC: Semantic-aware model checking for fast discovery of deep bugs in cloud systems. In Proceedings of the 11th USENIX Conference on Operating Systems Design and Implementation, OSDI '14, pages 399-414, USA, 2014. USENIX Association

[34] Mengxing Liu, Mingxing Zhang, Kang Chen, Xuehai Qian, Yongwei Wu, Weimin Zheng, and Jinglei Ren. DudeTM: Building Durable Transactions with Decoupling for Persistent Memory. In Proceedings of the 22nd International Conference on Architectural Support for Programming Languages and Operating Systems, ASPLOS '17, pages 329-343, 2017

[35] Qingrui Liu, Joseph Izraelevitz, Se Kwon Lee, Michael L Scott, Sam H Noh, and Changhee Jung. iDO: Compiler-Directed Failure Atomicity for Nonvolatile Memory. In Proceedings of the 51st Annual IEEE/ACM International Symposium on Microarchitecture, MICRO '18, pages 258-270, 2018.

[36] Sihang Liu, Korakit Seemakhupt, Yizhou Wei, Thomas Wenisch, Aasheesh Kolli, and Samira Khan. Cross-failure bug detection in persistent memory programs. In Proceedings of the 25th International Conference on Architectural Support for Programming Languages and Operating Systems, ASPLOS '20, pages 1187-1202, New York, NY, USA, 2020. Association for Computing Machinery.

[37] Sihang Liu, Yizhou Wei, Jishen Zhao, Aasheesh Kolli, and Samira Khan. PMTest: A fast and flexible testing framework for persistent memory programs. In Proceed ings of the 24th International Conference on Architectural Support for Programming Languages and Operating Systems, ASPLOS '19, pages 411-425, New York, NY, USA, 2019. Association for Computing Machinery.

[38] Changwoo Min, Sanidhya Kashyap, Byoungyoung Lee, Chengyu Song, and Taesoo Kim. Cross-checking semantic correctness: The case of finding file system bugs. In Proceedings of the 25th Symposium on Operating Systems Principles, SOSP '15, pages 361-377, 2015

[39] Jayashree Mohan, Ashlie Martinez, Soujanya Ponnapalli, Pandian Raju, and Vijay Chidambaram. Finding crash-consistency bugs with bounded black-box crash testing. In Proceedings of the 13th USENIX Conference on Operating Systems Design and Implementation, OSDI '18, pages 33-50, 2018.

[40] Madan Musuvathi, Shaz Qadeer, and Thomas Ball. CHESS: A systematic testing tool for concurrent software. Logic-Based Program Synthesis and Transformation, page 16, November 2007.

[41] Madanlal Musuvathi, Shaz Qadeer, Thomas Ball, Gerard Basler, Piramanayagam Arumuga Nainar, and Iulian Neamtiu. Finding and reproducing Heisenbugs in concurrent programs. In Proceedings of the 8th USENIX Conference on Operating Systems Design and Implementation, OSDI '08, pages 267-280, 2008.

[42] Ian Neal, B. Reeves, Ben Stoler, Andrew Quinn, Youngjin Kwon, S. Peter, and Baris Kasikci. AGAMOTTO: How persistent is your persistent memory application?
In Proceedings of the 14th USENIX Conference on Operating Systems Design and Implementation, OSDI '20, 2020.

[43] Azalea Raad, John Wickerson, Gil Neiger, and Viktor Vafeiadis. Persistency semantics of the Intel-X86 architecture. Proceedings of the ACM on Programming Languages, 4(POPL), December 2019.

[44] Cindy Rubio-González, Haryadi S. Gunawi, Ben Liblit, Remzi H. Arpaci-Dusseau, and Andrea C. Arpaci-Dusseau. Error propagation analysis for file systems. In Proceedings of the 30th ACM SIGPLAN Conference on Programming Language Design and Implementation, PLDI '09, pages 270-280, 2009.

[45] Olli Saarikivi, Kari Kähkönen, and Keijo Heljanko. Improving dynamic partial order reductions for concolic testing. In 2012 12th International Conference on Application of Concurrency to System Design, pages 132-141. IEEE, 2012.

[46] Koushik Sen and Gul Agha. Automated systematic testing of open distributed programs. In Proceedings of the 9th International Conference on Fundamental Approaches to Software Engineering, FASE'06, pages 339-356. Springer, 2006.

[47] Koushik Sen and Gul Agha. A race-detection and flipping algorithm for automated testing of multi-threaded programs. In Proceedings of the 2nd International Haifa Verification Conference on Hardware and Software, Verification and Testing, HVC'06, pages 166-182. Springer, 2006.

[48] Helgi Sigurbjarnarson, James Bornholt, Emina Torlak, and Xi Wang. Push-button verification of file systems via crash refinement. In Proceedings of the 12th USENIX Conference on Operating Systems Design and Implementation, OSDI '16, pages 1-16, 2016.

[49] Samira Tasharofi, Rajesh K Karmani, Steven Lauterburg, Axel Legay, Darko Marinov, and Gul Agha. TransDPOR: A novel dynamic partial-order reduction technique for testing actor programs. In Proceedings of the 14th foint IFIP WG 6.1 International Conference and Proceedings of the 32nd IFIP WG 6.1 International Conference on Formal Techniques for Distributed Systems, FMOODS'12/FORTE'12, pages 219-234. Springer, 2012.

[50] H. S. P. Wong, H. Lee, S. Yu, Y. Chen, Y. Wu, P. Chen, B. Lee, F. T. Chen, and M. Tsai. Metal-oxide RRAM. Proceedings of the IEEE, 100(6):1951-1970, June 2012.

[51] H. S. P. Wong, S. Raoux, S. Kim, J. Liang, J. P. Reifenberg, B. Rajendran, M. Asheghi, and K. E. Goodson. Phase change memory. Proceedings of the IEEE, 98(12):22012227, December 2010.

[52] W. Xu, H. Moon, S. Kashyap, P. Tseng, and T. Kim. Fuzzing file systems via two-dimensional input space exploration. In 2019 IEEE Symposium on Security and Privacy (S\&P), pages 818-834, 2019.

[53] Junfeng Yang, Can Sar, and Dawson Engler. Explode: A lightweight, general system for finding serious storage system errors. In Proceedings of the 7th USENIX Symposium on Operating Systems Design and Implementation, OSDI '06, page 10, USA, 2006. USENIX Association.

[54] Junfeng Yang, Paul Twohey, Dawson Engler, and Madanlal Musuvathi. Using model checking to find serious file system errors. ACM Transactions on Computer Systems, 24(4):393-423, November 2006.

[55] Hanbin Yoon, Justin Meza, Naveen Muralimanohar, Norman P. Jouppi, and Onur Mutlu. Efficient data mapping and buffering techniques for multilevel cell phasechange memories. ACM Transactions on Architecture and Code Optimization, 11(4):40:1-40:25, December 2014

[56] Naling Zhang, Markus Kusano, and Chao Wang. Dynamic partial order reduction for relaxed memory models. In Proceedings of the 36th ACM SIGPLAN Conference on Programming Language Design and Implementation, PLDI '15, pages 250-259, 2015. 